\title{
COMPARATIVE INVESTIGATION OF MECHANICAL-PHYSICAL CHARACTERISTICS OF BIODEGRADABLE AND NON-DEGRADABLE YARNS
}

\author{
Kira Krikštanavičienė*, Sigitas Stanys, Vaida Jonaitienė \\ Kaunas University of Technology, Faculty of Mechanical Engineering and Design, Department of Material Engineering, \\ Lithuania, Studentu st. 56, LT - 51424 Kaunas \\ E-mail kira.krikstanaviciene@stud.ktu.lt
}

\section{Abstract:}

This article presents the results from investigations of tensile tests, absorbency test and degradation test of biodegradable and non- or partly biodegradable yarns produced from pure poly hydroxybutyrate-co-valerate (PHBV), poly (lactide acid) (PLA), isotactic polypropylene (iPP) polymers and their blends. The results indicate that mechanical-physical properties of PHBV are improved by adding PLA and iPP to PHBV. The main results indicate that the PHBV/PLA and PHBV/iPP (70/30) blends had better mechanical properties than pure PHBV, as well as improved immiscibility and the same or lower degradation in sodium chloride solution, respectively. The PHBV/PLA and PHBV/iPP blends showed a tendency for lower crystallinity and stiffness of the yarns, rendering them less stiff and fragile. The absorption tests showed that absorption dynamic process depends on the structure and raw materials of the yarns. The disinfectant in all samples is absorbed faster than blood. Research results showed that pure PHBV yarns have good hydrophobic properties, compared with pure PLA and iPP yarns. The use of additional PLA and iPP polymers changed the wetting behaviour of yarns. Absorption time of blended yarns in disinfectant liquid decreases and absorption time in the case of blood significantly increases in comparison with PLA and iPP yarns and decreases compared with PHBV yarns. The degradation tests (within 90 days in a solution of sodium chloride) showed that pure PHBV and PHBV/PLA blends degraded at different rates but with the loss of the same weight, while pure PHBV and PHBV/iPP blends degraded at the same rate, but PHBV/iPP blends had worse destruction results. Such improvements are expected to be important for the practical application of PHBV in some fields.

\section{Keywords:}

Polyhydroxybutyrate-co-valerate, polylactid acid, polypropylene, extrusion, mechanical properties

\section{Introduction}

There are a number of polymers with widely differing properties, making them useful for different applications. They can either be used as a single polymer or by blending copolymer or polymer granules separately. Blends of polymers with other kinds of polymers can be used to improve their physical and mechanical properties. Blends can also help in the development of new low-cost products with better performance.

Plastics are widely used because of low cost, easy fabrication into complex shapes, light weight and excellent performance, and include soft rubbers to fibres stronger and stiffer than steel. The main advantage of synthetic polymers/plastics is that their chemical structure is relatively simple and more heat stable than the natural counterparts.

Polypropylene (PP) is a synthetic material most often used in various fields of textile. Isotactic polypropylene (iPP) is a typical thermoplastic with a number of desirable basic properties making it a versatile material among thermoplastics. PP yarns are now used in medical practice as PP resists the action of microorganisms, is inert relative to live tissue and is chemically stable; in addition,
PP yarn possesses good mechanical properties [1-3]. These properties together with their low specific gravity and low cost are the primary reasons for its popularity as a commodity resin. The synthetic plastics are resistant to destruction, and biotechnological polymers such as poly (3-hydroxybutirate) (PHB) and poly (lactide acid) (PLA) are alternatives to petroleum-based thermoplastics and represent an interesting and growing market [4].

Homopolymer PHB and co-polyester poly (hydroxybutyrateco-valerate) (PHBV) are the best known materials of polyhydroxyalkanoate (PHA) family. PHAs are aliphatic thermoplastic polyesters directly produced by numerous microorganisms through bacterial fermentation of sugars or lipids. Their mechanical and thermal properties span a wide range, including materials reminding PP and others that are elastomeric. In the main, its properties depend on copolymer composition. These polymers are non-toxic and renewable [5-8].

Tissue engineering or suturing are the current areas for applications of biodegradable polymers in medicine. Mechanical properties and time of degradation must match application needs. The general criteria of selection of polymer for medical applications are: sufficiently strong until the healing 
of surrounding tissue; does not invoke inflammatory or toxic response; is metabolised in the body after fulfilling its purpose, leaving no trace; be easily processable into the final product form; must demonstrate acceptable shelf life; and be easily sterilised $[9,10]$.

The main advantages are: good biocompatibility; possibility of changing in composition and in physical-mechanical properties; low friction coefficients; easy processing and workability; ability to change surface chemically and physically; and ability to immobilise cells or biomolecules within them or on the surface. The main disadvantages are: presence of substances that may be issued in the body monomers (toxic), catalysts, additives after degradation; easy water and biomolecules absorption from surrounding; and low mechanical properties. The final properties of a material depend on both intrinsic molecular structure of the polymer and chemical and mechanical processes undergone $[9,10]$.

PHB polymer has many technical drawbacks limiting its applications. Low rate of crystallisation related to the low heterogeneous nucleation density; and relatively stiff chain, short chain segment, and, consequently a high glass transition temperature $\mathrm{Tg}$, approximately $10^{\circ} \mathrm{C}$, are the drawbacks [11-13].

Upon extrusion of PHB homopolymers, the molded parts have not completed crystallisation yet result in pronounced after-molding ageing effects, and secondary crystallisation resulting in brittle products. Also PHB is thermally unstable resulting in a very narrow processing window. Serious degradation due to random chain scission occurs during melt processing $[11,14]$. The copolymerisation of $\mathrm{PHB}$ entities with 3-hydroxyvalerate (3HV), 4-hydroxybutyrate and hydroxyhexanoate co-monomers modifies the physical and mechanical characteristics of the parent $\mathrm{PHB}$, such as ductility and toughness, to depress its processing temperature and embrittlement. PHB, PHBV and other copolymers are biodegradable and biocompatible $[5,15,16]$.

PHBV copolymer can exist with various mole percentages of $3 \mathrm{HV}$. Incorporation HV in the structure of PHB changes the mechanical properties of the polymer. $\mathrm{PHB}$, being isodimorphic, accommodates the $3 \mathrm{HV}$ units into its crystal structure and, hence, addition of $3 \mathrm{HV}$ does not have a huge impact on the crystallinity of PHB [17]. The addition of $3 \mathrm{HV}$ makes the polymer more flexible and reduces the strength (elongation is $2.25 \%$, melting temperature is $153^{\circ} \mathrm{C}$, crystallinity is $58.7 \%$ and glass transition temperature is $6^{\circ} \mathrm{C}$ ). This also makes PHBV easier to process than PHB.

From the view of improving and modifying the properties of materials made from PHBV, in particular, their mechanical properties can be blended with various biodegradable or non-degradable polymers. Blending of such polymers has been widely studied [18-22]. Maekawa et al. [19] reported that blends of $\mathrm{PHB}$ and cellulose propionate were completely miscible since they had a single glass transition, a depression in the equilibrium melting temperature of $\mathrm{PHB}$ and a decrease in the spherulitic growth rate of the PHB component. Also, the tensile strength was better for the blend PHB/cellulose propionate than for PHB only $[17,20]$.

However, not all blends are compatible. For example, PHB and poly (L-lactide) (PLLA) form a compatible mixture only when the molecular weight of PLLA is smaller than 11700 $\mathrm{Da}$ [23]. The reason for choosing PLA polymer for our research work can be explained from the point of view that among many biodegradable polymers, PLA is a material used in many biomedical applications. It can also be used in different engineering applications due to its bio-based origin, reasonable strength, transparency and pliability. Its mechanical properties are considered to be broadly similar to those of conventional polyethylene terephthalate (PET), and, probably due to its lower melting and softening temperatures, comparisons with $\mathrm{PP}$ are also appropriate. Although, PLA is biocompostable, with a low $\mathrm{Tg}$ (around $55^{\circ} \mathrm{C}$ ) and low crystallisation rate. PLA becomes soft when the temperature approaches its $T g$ and its E-modulus cannot be backed up by crystallinity because PLA generally is amorphous after processing [24-27].

Most of the studies on PLA blended with PHAs that have been reported in the literature concern the materials prepared by solvent casting [28-31]. The majority of the blends were found to be immiscible, except those with the molecular weight of PLA [29] or of PHB [30] was very low. Relatively few studies have been conducted on the preparation and characterisation of PLA/PHA blends by melt mixing despite the fact that both polymers are thermoplastic and their processing is similar to the one of classical oilbased polymers. Noda et al. [32] blended Nodax ${ }^{\mathrm{TM}}$ with PLA. This bacterially produced PHA copolymers comprising 3-hydroxybutyrate and other 3-hydroxyalkanoate units (PHBHHx) improved the toughness of the blends when added at a level below 20 wt.\%. Zhang and Thomas [33] and Gerard, T. et al [34] studied PLA/PHB and PLA/PHBV, respectively, prepared by melt compounding. They found that the blends were immiscible for all compositions.

In our previous research [35], the attempts to produce pure PHBV multifilament yarns using single screw extruder equipment were made. It was found that PHBV copolymer is very sensitive to temperature changes. The obtained yarn structure was more similar to the monofilament than to multifilament. The produced samples were also not strong.

The present paper reports the results of an exploratory experimental investigation to study yarns produced from PHBV, PLA and iPP polymer granules and their PHBV/PLA and PHBV/iPP blends. PLA and iPP polymers were chosen as the support material in order to obtain a multifilament PHBV yarn structure.

The aim is to develop new completely degradable and partially degradable multifilament structures with better mechanical and physical properties that cannot be achieved with only homo or copolymers. Mechanical and physical behaviours were studied depending on the multifilament composition applied melt spinning process. 


\section{Experimental}

\subsection{Materials}

Multifilament yarns produced from PHBV copolymer granules with $8 \%$ of valerate (Nature Plast, France), PLA 6201D granules (Nature Works, USA) and iPP Moplen HP462R granules (Basell Service B. V., Germany) and their blends were the experimental materials used in this study. The main characteristics of polymers (given by provider) are presented in Table 1.

Blended yarn samples were prepared from polymer blends PHBV/PLA, PHBV/PP at a proportion of 70:30. PHBV, PLA and iPP polymer granules of this type can be used for producing yarns and are suitable for extrusion applications.

\subsection{Multifilament yarns preparation}

Melt spinning of all yarns was carried out using COLLIN ${ }^{\circledR}$ CMF 100 (Dr. Collin GmbH, Germany) laboratory single screw extruder $(L / D=25: 1$ ) equipment (Figure 1 ). This extruder includes seven heating zones where the temperatures are independently and gradually fixed from $10^{\circ} \mathrm{C}$ up to $300^{\circ} \mathrm{C}$. Four godets are driven separately in a speed range of $50-800 \mathrm{~m} /$ min. The yarns were extruded individually before the actual yarn preparation in order to understand the behaviour of the yarns under actual running conditions and to select the range of process parameters that run the yarns on the machine.

Multifilament yarns from the polymers were formed at identical melt spinning parameters. The conditions of formation and other technical details are given in Table 2.
Molten polymer passes through spinneret consisting of 24 circular holes with a diameter of $0.45 \mathrm{~mm}$ each to produce a multifilament continuous yarn. Cooling of the resultant filaments was achieved with cross-flow air quenching at a temperature of $12^{\circ} \mathrm{C}$. The multifilament is covered with an appropriate spin finish (oil emulsion) ensuring the monofilament cohesion along the process, rolled up on four heated rolls with varying speeds (Table 2) in order to ensure the optimal drawing. The speed of rolls was fixed from $99 \mathrm{~m} / \mathrm{min}$ to $250 \mathrm{~m} / \mathrm{min}$. The as-spun filaments were drawn with an overall draw ratio of 2.525 in a continuous spin drawing process.

Table 2. Parameters for spinning step to obtain multifilament yarns.

\begin{tabular}{|c|c|c|}
\hline \multicolumn{2}{|l|}{ Parameter } & Unit \\
\hline \multicolumn{3}{|l|}{ Extrusion } \\
\hline \multicolumn{2}{|c|}{ Extruder 7 zones temperature $T,{ }^{\circ} \mathrm{C}$} & 188 \\
\hline \multicolumn{2}{|c|}{ Screw speed, rpm } & 20 \\
\hline \multicolumn{2}{|l|}{ Extruder pressure $P, \mathrm{MPa}$} & 3.2 \\
\hline \multicolumn{2}{|l|}{ Melt pump speed, rpm } & 13 \\
\hline \multicolumn{2}{|l|}{ Hole diameter, $\mathrm{mm}$} & 0.45 \\
\hline \multicolumn{2}{|l|}{ Hole length, $\mathrm{mm}$} & 1.3 \\
\hline \multicolumn{3}{|l|}{ Spinning } \\
\hline \multirow{4}{*}{$\begin{array}{l}\text { Stretching rolls temperature, } \\
{ }^{\circ} \mathrm{C} / \text { speed, rpm }\end{array}$} & No 1 & $69 / 99$ \\
\hline & No 2 & $75 / 198$ \\
\hline & No 3 & $78 / 228$ \\
\hline & No 4 & $85 / 250$ \\
\hline \multicolumn{2}{|l|}{ Drawing ratio } & 2.525 \\
\hline
\end{tabular}

Table 1. The main properties of PHBV, PLA, and iPP polymers, given by providers.

\begin{tabular}{|c|c|c|c|c|}
\hline & $\begin{array}{l}\text { Glass transition } \\
\text { temperature, }{ }^{\circ} \mathrm{C}\end{array}$ & Melting temperature, ${ }^{\circ} \mathrm{C}$ & Melt flow index $\mathrm{g} / 10 \mathrm{~min}\left(230^{\circ} \mathrm{C} / 2.16 \mathrm{~kg}\right)$ & $\begin{array}{c}\text { Density } \\
\text { g/cm }\end{array}$ \\
\hline PHBV & 5 & $165-175$ & $15-30$ & 1.24 \\
\hline PLA & $55-60$ & $220-240$ & $15-30$ & 1.08 \\
\hline iPP & 11 & $160-171$ & 25 & 0.91 \\
\hline
\end{tabular}

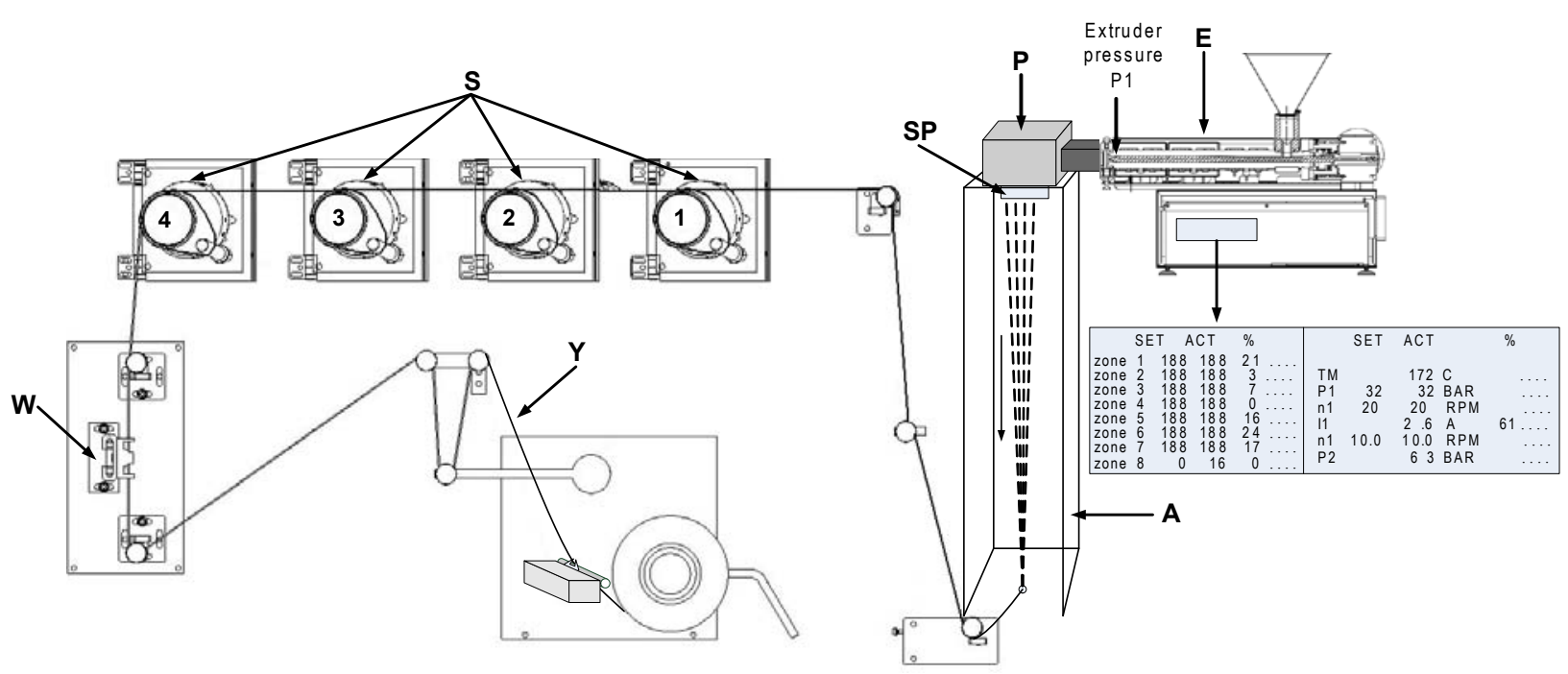

Figure 1. Principal scheme of the spinning setup "COLLIN CMF 100": $E$ - extruder, $P$ - melting pump, SP - spinneret, A - air quench cabinet, $S$ stretching godets (No 1, No 2, No 3, and No 4), W - whirling unit, $Y$ - yarn. 


\subsection{Mechanical and physical properties}

\subsubsection{Tensile tests}

Tensile tests were made under standard conditions, i.e. at relative humidity $\varphi=65 \pm 5 \%$ and temperature $T=20 \pm 2^{\circ} \mathrm{C}$ (ISO 139). The measurements were taken after 7 days of fabrication of the samples. The yarn linear density was determined using Zweigle L232 reeling machine. To determine the linear density of yarn, five specimens of $200 \mathrm{~m}$ yarn length were prepared and the average value was determined according to ISO 2060:1994.

Mechanical properties of the yarns were evaluated according to ISO $2060: 1993$ by the tensile tests with a tensile testing machine Zwick/Z005 and testXpert ${ }^{\circledR}$ software. The test conditions were the following: specimen gauge length $250 \mathrm{~mm}$, crossbar speed $500 \mathrm{~mm} / \mathrm{s}$ for all tested yarns and pre-tension $0.5 \mathrm{cN} /$ tex. Yarns were stretched till breaking point.

\subsubsection{Absorbency tests}

The wettability of yarns can be characterised by contact angle. The testing methods for contact angle can be divided into two categories: the dynamic drop micro-observation [36] and the dynamic testing method with L. Wilhelmy plate. The static testing drop method was used in this study.

Absorbency tests by drop method were made using two types of liquids: blood (Type 0+) and disinfectant (Cutasept F, AS Chemi-Pharm, Estonia). The blood-donating patient was healthy in terms of blood parameters with no indication of blood-related disease. This test method is designed to measure liquid absorbency of yarns by measuring the time it takes for a drop of liquid placed on the yarn surface to be completely absorbed into the yarn. A drop of $\sim 0.005 \mathrm{~g}$ is made on the yarn surface from a height of $0.2 \mathrm{~cm}$ with the needle. A stop watch is started as soon as the drop falls on the yarn and is stopped as soon as the image of the reflected light disappears at the edge of the drop, i.e. the liquid drop is completely absorbed by the yarn or variation is irrelevant. This is termed as drop absorbency time. The process is recorded by stereo microscope (Nikon Stereoscopic Microscope SMZ 800 ) and digital camera Nikon Coolpix 4500 connected to a computer (Figure 2). The changes in fluid angles in time were measured using the Lucia Image 5.0 program, and compared with the initial wetting angles at the initial observation time of $0 \mathrm{sec}$. The results were obtained from 10 individual measurements.

The absorbency depends on the liquid surface tension. The surface tension measurements of fluids used in the experiments were carried out by DataPhysics DCAT21 tensiometer. Surface tension measurements of used liquids were selected by dynamic L. Wilhelmy plate method (DIN 53915, ASTMc-971). Immediately after donation, the anticoagulant agent $(3.8 \%$ sodium citrate) was added to the blood sample of $20 \mathrm{ml}$ and it was then transferred to the experimental laboratory and underwent surface tension measurement procedure.

\subsubsection{Degradation tests}

Five specimens of each yarn type with dimensions of $100 \mathrm{~mm}$ length and the same weight, incubated in $400 \mathrm{ml}$ of sodium chloride of $0.9 \%$ solution (Fresenius Kabi, Poland) at $37^{\circ} \mathrm{C}$, were prepared for degradation studies. Sodium chloride was in blood plasma and tissue fluids of body. It is the most important inorganic component supporting the osmotic pressure of blood plasma and extracellular fluid. Sodium chloride of solution $0.9 \%$ was used to replace lost body fluids and salts. Other medicines that are given by injection or by a drip diluted with sodium chloride solution of $0.9 \%$ can also be used as a sterile irrigation solution. Sodium chloride solution of $0.9 \%$ is a clear, colourless solution with nominal ph $=5.5$ [37].

Temperature of $37^{\circ} \mathrm{C}$ was chosen because it is close to human body temperature. Reservoirs with sodium chloride solution were placed into water bath with thermostat device (Lauda Ecoline E100, 011, Germany) at $37^{\circ} \mathrm{C}$. The sodium chloride solution was changed every three days. The specimens were taken out, washed with distilled water and dried in laboratory at the end of each immersion period under standard conditions (relative humidity $\varphi=63 \pm 5 \%$, temperature $T=20 \pm 2^{\circ} \mathrm{C}$ for 24 hours).

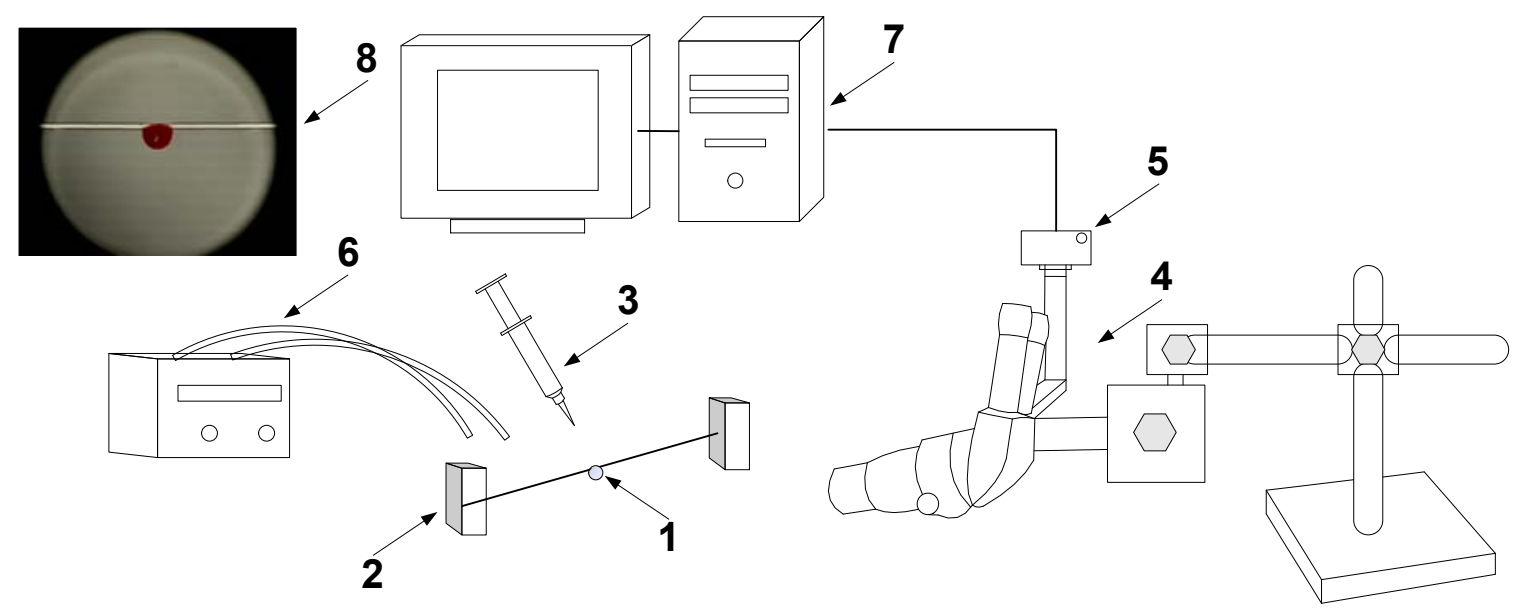

Figure 2. Principal scheme of absorbency test: 1) drop of liquid on the yarn; 2) yarn anchorage system; 3) pipette; 4) stereoscopic microscope; 5) digital camera; 6) light source; 7) computer; 8) picture of video record. 
The yarn specimens were weighed before the test. Degradation was measured every 10 days three months by measuring the mass loss. The weight loss $\left(W_{l}\right)$ of each yarn sample, taken as an indication of biodegradability, was calculated as follows [11]:

$$
W_{l}(\%)=\frac{\left(W_{m}-W_{0}\right)}{W_{0}} \cdot 100
$$

where $W_{0}$ is the original sample weight and $W_{m}$ is the measured sample weight after burial in sodium chloride. At the end of the investigation, mechanical properties of the samples were evaluated according to ISO 2060:1993 by the tensile tests with a tensile testing machine Zwick/Z005 and testXpert $₫$ software.

\section{Results and discussion}

The results reported in our previous study demonstrate that bacterial plastics (PHB - 8\% HV) have some weaknesses that need to be addressed. The aim of previous research was to produce multifilament yarns from pure $\mathrm{PHB}-8 \% \mathrm{HV}$ copolymer granules by extrusion equipment COLLIN CMF - 100. The study showed that the obtained yarns structure is more similar to monofilament than to multifilament structure because all 24 filaments were stuck together. This was explained by the insufficient temperature of cross-flow air in quench cabin to cool down the filaments. Other filament cooling techniques should be used here.

Modification of PHB - 8\% HV polymer can normally inform the expected results. Blending of PHBV-based materials with other polymers is an effective and economic way to regulate their properties. Chemical and/or physical modifications are commonly adopted in order to improve their properties. In this study, physical modification blends with natural PLA and synthetic iPP polymers and type of PHB - 8\% HV copolymer were performed. The addition of $30 \%$ of PLA and iPP polymer granules improved the processability of $\mathrm{PHB}-8 \% \mathrm{HV}$.

\subsection{Tensile tests}

The tensile properties of filaments are strongly influenced by their physical structure that is controlled by the choice of the starting material and the fibre formation conditions. Table 3 lists the mean values of linear density and tensile characteristics of extruded yarns. The stress-strain curves of drawn multifilament yarns are shown in Figure 3.

It was established that iPP (C3) yarns are the strongest and can withstand the most stress per linear density $(18.14 \mathrm{cN} /$ tex). The breaking tenacity of pure PLA (C2) yarns is $10.52 \mathrm{cN} /$ tex and the lowest for pure PHBV (C1) yarns is only $6.50 \mathrm{cN} /$ tex. Neat PHBV yarn was rigid and brittle; this result is in line with our earlier evidence of PHBV yarns. The breaking force of PHBV was improved dramatically to $73.08 \%$ and $42.77 \%$ respectively after the addition of $30 \%$ PLA and iPP, indicating that the fracture behaviour in the tensile test changed from a brittle fracture of neat PHBV to a ductile fracture of the blend. The PHBV/iPP (C5) and PHBV/PLA (C4) yarns have slightly differing values of breaking tenacity (as 9.28 and $11.25 \mathrm{cN} / \mathrm{tex}$ ).
Furthermore, the tenacity-extension curves were elaborated for each yarn where the stress-strain behaviour of the used materials can be observed. It is well known that higher crystalline is for a polymer, higher are the tensile modulus and the tensile strength and lower is the elongation at break. Tenacity-extension curve evaluation (Figure 1) revealed that C1 curve of PHBV yarn is brittle and is also a little strong because there is some strain in a high stress. The fracture of a brittle material is sudden with a little or no plastic deformation. C4 (PHBV/PLA) is a second yarn under the strength that is not very ductile compared with C3 (iPP) curve. The tensile strength of $\mathrm{C} 4$ yarn was quite close to that of $\mathrm{C} 2$. However, the elongation at break of C4 (34.20\%) was markedly lower than that of C2 (146.90\%), although the tensile modulus was higher.

The C3 curve showed that iPP yarn is the strongest $(18.14 \mathrm{cN} /$ tex) and is very ductile (279.00\%). Therefore, it requires more work at the maximum force $(2.843 \mathrm{~J})$. An amount of $30 \%$ of iPP granules in mixture (curve $\mathrm{C5}$ ) twice decreases the elongation at break and the tensile strength, increases the tensile modulus $11.19 \%$, and the work of break is reduced to $65.49 \%$ compared with C3 yarns and increased to $101.22 \%$ compared with C1 samples. Blended PHBV/PLA yarns showed a considerably higher level of strength at a lower elongation at break than other yarns. The blend yarns of PHBV/PLA showed a slight increase in the tensile strength $(11.25 \mathrm{cN} /$ tex $)$ and significant increase in tensile modulus (102.73 cN/tex) that result in a stiffer yarn. Differences in the crystallisation and orientation behaviour during the structure formation can be considered as the cause.

Note that the proportion of $30 \%$ of iPP and PLA reduced the stiffness and fragility of PHBV in the PHBV/iPP and PHBV/ PLA blends, causing the tensile strain at break to increase, respectively, increase the work of break and tensile modulus, and tensile strength to decrease. These results suggest a plasticiser effect resulting from the presence iPP and PLA in the PHBV, which was confirmed by the increase in tensile strength. PLA and iPP content as plasticisers does help in getting a multifilament structure and more flexible yarn. From the experiments, it can be noted that the blended yarn with

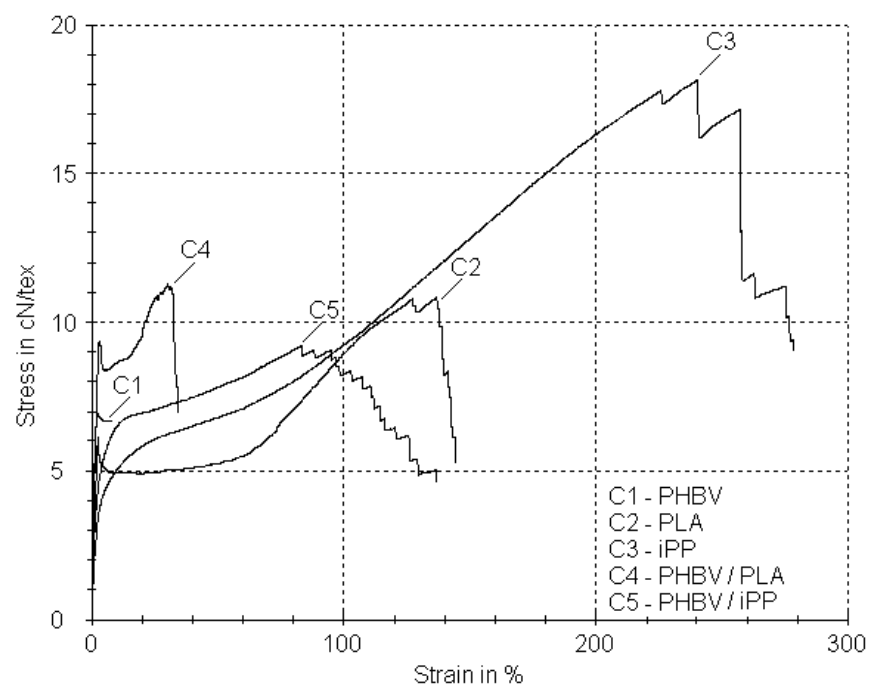

Figure 3. Tenacity-extension curve for: PHBV, PLA, iPP, PHBV/PLA and PHBV/iPP yarns. 
$30 \%$ of PLA has good mechanical characteristics compared with other yarns.

PHBV was stiffer and more brittle than iPP. iPP can improve the tensile toughness of PHBV, but is not biodegradable. iPP in comparison with PLA may be a good alternative. The physical properties and structure of PLA have been studied by several researchers [27] and these works confirmed that this polymer has significant commercial potential as a textile fibre. Its mechanical properties are considered to be broadly similar to those of conventional PET, and, probably due to its lower melting and softening temperatures, the comparisons with iPP are also appropriate. However, PHBV blends well with PLA, and with their load elongation curves being very similar, this would enable the full properties of both fibres to be exploited.

From the data presented (Table 3), it can be noticed that the liner density of the yarns is different. The linear density values cannot be compared, because yarns are made from different raw materials. From our previous research [35], it was revealed that a low linear density of PHBV yarns could be obtained at higher polymer melting temperature $\left(188^{\circ} \mathrm{C}\right)$ combined with low extruder pressure (3.2 $\mathrm{MPa})$. However, this temperature is the maximum, which can be used for the production of pure $\mathrm{PHBV}$. In this study, it was reconfirmed that the obtained $\mathrm{C} 1$ yarn structure and mechanical characteristics were not perfect. As has already been mentioned, the decision was taken to improve the structure of the PHBV polymer mixing with PLA and iPP polymers. Analyzing the data from Table 4, it was found that using the current extrusion parameters C1 samples (19 tex) have the smallest value of linear density. Comparing the density values of the pure yarns, C2 (PLA) yarns (53.69 tex) have the maximum linear density. Difference between samples $\mathrm{C} 1$ and C2 is $182.57 \%$, and the difference between the samples C1 and $\mathrm{C} 3$ is $136.03 \%$. Comparing the blended yarns, the results showed that $30 \%$ of PLA polymer increased the linear density of C4 yarn to $99.79 \%$ (38.14 tex), while the $30 \%$ of iPP polymer increased the linear density of C5 yarn to $203.87 \%$ (58.01 tex). It is the highest value of linear density produced of yarns.

However, as the analysis of mechanical properties has shown, the blended yarns have a stable structure, which is not so sensitive to variations of temperature, and have better mechanical properties, which means trying to change the manufacturing parameters (increase melting temperature, drawing ratio, speed of stretching rolls, etc) in order to get thinner yarns with improved characteristics.

\subsection{Absorbency tests}

Many scientists emphasise the importance of absorption; therefore, this phenomenon is continuously studied. The liquid droplet spread in multifilament yarns is not widely examined depending on the observation time and type of liquid. Absorption, conduction properties and moisture transfer in the final product are the important factors relevant for medical textiles. Therefore, it is important to determine the absorption properties of yarns depending on the yarn structure parameters.

Table 4 lists the values of surface tension for different experimental liquids. The obtained data show that the surface tension of blood is higher than of the disinfectant solution.

As has already been mentioned, behaviour of liquid drops on the yarns was recorded. The force manifestation of cohesiveacting disinfectant drop was recorded during the experiment. This has led to rapid changes in the drop configuration occurring during the first seconds of the experiment. Consequently the angles were measured: the first $10 \mathrm{sec}$ is $1-\mathrm{sec}$ interval, and then every $20 \mathrm{sec}$, until the liquid is completely absorbed or liquid distribution is irrelevant.

The pictures obtained during each experiment provided information about the values of the right and left corners of the drops. The results of studies of the average value of right and left contact angles of liquids drop with each yarn are shown in Figure 4. It was seen that the investigated liquid behaviour was different (Figure 4) and disinfectant's absorption was faster than blood when the contact angle values are examined. The values of contact angles of liquid drops on yarns at selected time intervals are provided in Table 5.

At the initial time, blood drops contacting angle with yarn have greater angles $\left(0.32^{\circ}-17.15^{\circ}\right)$ than with the disinfectant (Table 5).

Table 4. Surface tension of the liquids used.

\begin{tabular}{|c|c|}
\hline Liquid & $\begin{array}{c}\text { Surface tension } \gamma(\mathrm{mN} / \mathrm{m}) \\
\pm \text { standard deviation } \mathrm{s}(\mathrm{mN} / \mathrm{m})\end{array}$ \\
\hline Disinfectant $\left(22^{\circ} \mathrm{C}\right)$ & $0.024 \pm 0.02$ \\
\hline Blood $\left(22^{\circ} \mathrm{C}\right)$ & $0.056 \pm 0.8$ \\
\hline
\end{tabular}

Table 3. Mechanical characteristics of yarns

\begin{tabular}{|c|c|c|c|c|c|}
\hline \multirow{2}{*}{ Characteristics } & \multicolumn{5}{|c|}{ Calculated value \pm coefficient of variation \% } \\
\cline { 2 - 6 } & PHBV - 100\% & PLA - 100\% & iPP - 100\% & PHBV (70)/PLA (30) & PHBV (70)/iPP (30) \\
\cline { 2 - 6 } & C1 & C2 & C3 & C4 & C5 \\
\hline Breaking force, cN/tex & $6.50 \pm 4.41$ & $10.52 \pm 4.76$ & $18.14 \pm 3.16$ & $11.25 \pm 5.07$ & $134.30 \pm 5.65$ \\
\hline Elongation at break, \% & $8.80 \pm 4.43$ & $146.90 \pm$ & $279.00 \pm 4.80$ & $34.20 \pm 6.13$ & $0.981 \pm 5.97$ \\
\hline Work of break, J & $0.012 \pm 4.43$ & $1.208 \pm 5.74$ & $2.843 \pm 5.85$ & $0.238 \pm 6.94$ & $26.27 \pm 6.89$ \\
\hline Tensile modulus, cN/tex & $239.44 \pm 5.84$ & $63.88 \pm 5.83$ & $23.33 \pm 5.48$ & $102.73 \pm 5.12$ & $58.01 \pm 4.99$ \\
\hline Linear density, tex & $19.09 \pm 5.42$ & $53.69 \pm 4.86$ & $45.06 \pm 3.49$ & $38.14 \pm 4.92$ & 5.30 \\
\hline
\end{tabular}




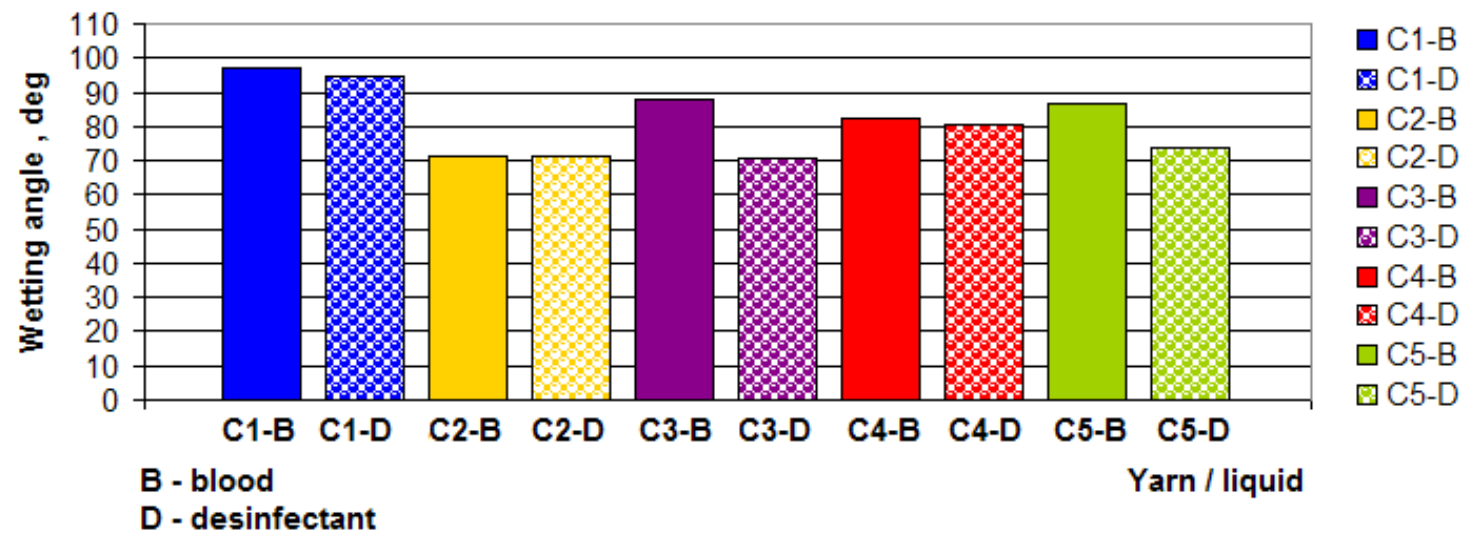

Figure 4. Average values of wetting angle for different experimental groups.

Table 5. The values of contact angles of liquid on yarns.

\begin{tabular}{|c|c|c|c|c|c|c|c|c|c|}
\hline \multicolumn{10}{|c|}{ Contact angle value $\theta$, deg \pm coefficient of variation, \% } \\
\hline & Liquid & Blood & Disinfectant & Blood & Disinfectant & Blood & Disinfectant & Blood & Disinfectant \\
\hline & Time & $0 \mathrm{sec}$ & $0 \mathrm{sec}$ & $10 \mathrm{sec}$ & $10 \mathrm{sec}$ & $60 \mathrm{sec}$ & $60 \mathrm{sec}$ & $120 \mathrm{sec}$ & $120 \mathrm{sec}$ \\
\hline \multicolumn{10}{|c|}{ Yarns } \\
\hline C1 & PHBV & $\begin{array}{c}96.87 \pm \\
10.02\end{array}$ & $\begin{array}{c}94.53 \pm \\
3.72\end{array}$ & $\begin{array}{c}86.92 \pm \\
5.37\end{array}$ & $\begin{array}{c}89.21 \pm \\
2.50\end{array}$ & $\begin{array}{c}85.39 \pm \\
5.11\end{array}$ & $\begin{array}{c}86.62 \pm \\
0.48\end{array}$ & $\begin{array}{c}83.52 \pm \\
5.20\end{array}$ & $\begin{array}{c}83.55 \pm \\
3.71\end{array}$ \\
\hline C2 & PLA & $\begin{array}{c}71.36 \pm \\
6.99\end{array}$ & $\begin{array}{c}71.04 \pm \\
0.02\end{array}$ & $\begin{array}{c}54.88 \pm \\
3.05\end{array}$ & $\begin{array}{c}61.39 \pm \\
2.68\end{array}$ & $\begin{array}{c}45.09 \pm \\
2.08\end{array}$ & - & $\begin{array}{c}35.52 \pm \\
3.87\end{array}$ & - \\
\hline C3 & iPP & $\begin{array}{c}87.63 \pm \\
0.33\end{array}$ & $\begin{array}{c}70.48 \pm \\
2.88\end{array}$ & $\begin{array}{c}84.98 \pm \\
0.56\end{array}$ & $\begin{array}{c}58.67 \pm \\
3.02\end{array}$ & $\begin{array}{c}83.44 \pm \\
0.69\end{array}$ & $\begin{array}{c}48.18 \pm \\
0.51\end{array}$ & $\begin{array}{c}81.40 \pm \\
0.33\end{array}$ & - \\
\hline C4 & PHBV/PLA & $\begin{array}{c}82.06 \pm \\
0.15\end{array}$ & $\begin{array}{c}80.64 \pm \\
3.44\end{array}$ & $\begin{array}{c}74.00 \pm \\
0.11\end{array}$ & $\begin{array}{c}67.59 \pm \\
2.24\end{array}$ & $\begin{array}{c}72.54 \pm \\
0.75\end{array}$ & $\begin{array}{c}53.82 \pm \\
2.86\end{array}$ & $\begin{array}{c}71.07 \pm \\
0.31\end{array}$ & $\begin{array}{c}34.43 \pm \\
1.49\end{array}$ \\
\hline C5 & PHBV/iPP & $\begin{array}{c}86.51 \pm \\
0.13\end{array}$ & $\begin{array}{c}73.97 \pm \\
5.93\end{array}$ & $\begin{array}{c}76.66 \pm \\
2.20\end{array}$ & $\begin{array}{c}62.96 \pm \\
0.69\end{array}$ & $\begin{array}{c}73.89 \pm \\
2.19\end{array}$ & $\begin{array}{c}32.72 \pm \\
2.49\end{array}$ & $\begin{array}{c}70.26 \pm \\
2.14\end{array}$ & - \\
\hline
\end{tabular}

Contact angles of disinfectant drop of $\mathrm{C} 2$ and $\mathrm{C} 3$ samples are the smallest $\left(71.04^{\circ}, 70.48^{\circ}\right.$, respectively). This could explain their intense absorption in yarn samples. Meanwhile, the other absorption angles formed with yarn were higher. Compared with yarns composed from pure polymers (C1, C2 and C3), the largest angle of the disinfectant drop was accounted with the $\mathrm{C} 1$ sample. Despite the low surface tension of disinfectant, further drop movement was smooth and not as intense as of the $\mathrm{C} 2$ and $\mathrm{C} 3$ samples. This is influenced by $\mathrm{C} 1$ yarn structure, which is more similar to monofilament than to a multifilament structure. The reason for such a yarn structure is presented in our previous study [35]. The absorption process of disinfectant lasts up to $\sim 1218 \mathrm{sec}$ from the initial to the last moment of $\mathrm{C} 1$ when the drop is fully absorbed. C4 yarn recorded a similar trend; the drop of disinfectant was absorbed faster in $\sim 114 \mathrm{sec}$ because this sample has a multifilament structure. Although the absorption process in $\mathrm{C} 2$ yarns is characterised by slow start, absorption processes are faster than in $\mathrm{C} 3$ yarn. It is assumed that iPP yarns are hydrophobic; however, in our study, the absorption depended on iPP yarn structure; multifilament yarns are non-textured and without finish. In the case of C3 yarns, the view [38] that the distribution of liquid occurs between the filaments, but does not soak into the filaments of yarn, was confirmed.
Comparing blended C4 (PHBV/PLA) and C5 (PHBV/iPP) yarns, C4 samples showed lower absorption results. As it can be seen from the data (Table 5, C4, C5 - $120 \mathrm{sec}$ ), the intensive prevalence is in C5 yarn. The analysis of the time to $10 \mathrm{sec}$ showed that the contact angle of C4 and C5 yarns compared with angles to 0 sec change increased $15.68 \%$ and $14.88 \%$, respectively. However, absorption process in C5 yarns (at time interval from $10 \mathrm{sec}$ to $60 \mathrm{sec}$ ) goes faster by $27.62 \%$ than $\mathrm{C} 4$ yarns.

It was difficult to ensure uniform droplet volume during the experiment, and so it had an effect on the size of the values of variation coefficient. Values of variation coefficient of disinfectant drop dynamic changes ranged from 0.02 to $5.93 \%$, while the values of variation coefficient of blood drop ranged from 0.11 to $10.02 \%$.

Blood has higher surface tension (Table 4) and higher viscosity (visual information) than disinfectant. The manifestation of spontaneous cohesive forces operating in drop of blood was recorded during the experiment. This resulted in rapid changes in the configuration of blood drop occurring within $10 \mathrm{sec}$ in C2 yarns $-26.19 \%$. Based on the data reported in Table 5 , the lowest contact angle made with yarns have $\mathrm{C} 2$ yarn $\left(71.36^{\circ}\right)$, 
was intense liquid (blood) absorption - $300.75 \mathrm{sec}$. As has been already mentioned, the yarn is not textured, and the filaments are simply clasped. Between the filaments are air spaces, moistened filaments transmitting the liquid faster in the vicinity filaments. So there is intense drop absorption on yarn surface; it can be reason why the liquid is absorbed much more quickly.

The contact angles of blood drop of the remaining samples $(\mathrm{C} 1, \mathrm{C} 3-\mathrm{C} 5)$ are relatively larger $\left(80^{\circ}\right)$. Compared with the contact angle results of $\mathrm{C} 1, \mathrm{C} 2$ and $\mathrm{C} 3$ yarns prepared from pure polymers, the C3 yarn (iPP) within the first 10 sec contact angle changes only $3.02 \%$ while the sample $\mathrm{C} 1$ changes $10.27 \%$. However, the absorption time of blood drop of $\mathrm{C} 1$ (PHBV) yarn is longest, even $3000 \mathrm{sec}$. The sudden changes in the contact angles can be explained via the human factor. Placing drops on the yarn it had to be attached to the yarn. At this moment a slight force was used, it was like pressing the drop, and so the drop seeks to recover its natural configuration.

Absorption time of blended yarns in disinfectant liquid case decreases $(41.25 \%)$ and in case of blood significantly increases $(81.18 \%)$ compared with PLA and iPP yarns and decreased (86.88\%, 29.91\%, respectively) compared with PHBV yarns. Here we observed similar trends, and the absorption process of C4 yarn is slower than of C5 sample. Obvious differences were seen between the experimental results of liquids. The disinfectant is absorbed faster than blood. Although the analysis of the contact angles of pure yarns showed that initially (first $10 \mathrm{sec}$ ) with drops of disinfectant the process is slower, only after 10-20 sec an intense spread of disinfectant was observed. The absorption process is reversed (C3 sample is an exception) with drops of blood on the pure yarn. During the first few seconds, sudden changes of contact angles of blood drop were triggered, and further intensity of absorption obviously decreases. Comparing the absorption intensity of the C4 and C5 blended yarns, it was observed that throughout the observation period $(120 \mathrm{sec})$ the absorption of blood was 43.91 and $36.98 \%$ slower, respectively.

The analysis indicates that the examined liquids absorbed a non-uniform speed of C2 and C3 yarns at different absorption observation intervals.
The process of disinfectant absorption process in pure yarns goes on from 79.25 to $1062.00 \mathrm{sec}$, and in blended yarns from 71.66 to $145.00 \mathrm{sec}$. The process of blood absorption in pure yarns goes on from 201.00 to $3000.00 \mathrm{sec}$ (change increased $282.48 \%$ ); in blended yarns it goes for $1800.00-1950.00 \mathrm{sec}$ (change increased $1344.82 \%$ ). In both cases, C1 yarn has good hydrophobic properties; this is seen as an evaluation of contact angle values of tested liquids. This is confirmed by the other authors that changes in absorbance lead to structure parameters of yarns [39]. The structure of C1 yarn is not multifilament, as already mentioned, and this may be one of reasons why the absorption process is slow. We received the biodegradable and partially degradable multifilament yarns with good hydrophobic properties compared with pure C2 (PLA) yarn samples by adding $30 \%$ of PLA and iPP granules. Yarns of pure PLA (C2) and iPP (C3) polymers have good sorption properties of material or structural features. In our case, from a medical point of view it is a negative characteristic because these yarns were orientated in the medical field of surgery.

\subsection{Degradation tests}

Many factors affect the rate of degradation of polymer-based products. They include the properties of the polymers, such as the molecular composition, the resulting intermolecular interactions, hydrophilic and hydrophobic behaviour, and the structural parameters, like degree of crystallinity, level of orientation, surface structure, and molecular weight.

As shown in Figure 5, the weight change values, calculated by equation (1) for all samples, show a loss in weight at the beginning of the sodium chloride test.

Degradation of yarns can be advantageously followed by measuring the change in tensile properties. The removal of polymer molecules or chain cleavage could account for a significant decrease in such properties. The few mechanical characteristics, tensile modulus, the breaking force and the elongation at break of samples degraded to different extent up to $\sim 20 \%$ of weight loss during the sodium chloride test, are reported in Table 6 . The tenacity curves of the yarns (Figure 6) were also submitted besides the data of mechanical characteristics.

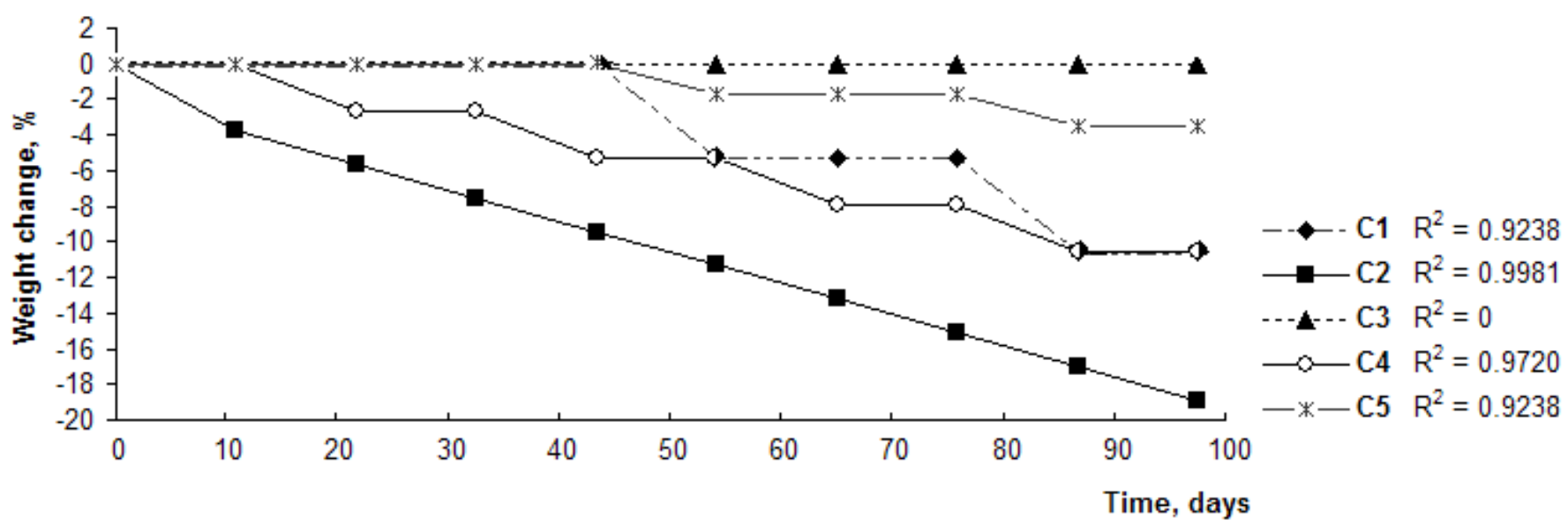

Figure 5. The weight change (\%) of yarns buried in sodium chloride as a function of time. 
Table 6. Mechanical characteristics of yarns after destruction test.

\begin{tabular}{|c|c|c|c|c|c|}
\hline \multirow{2}{*}{ Characteristics } & \multicolumn{5}{|c|}{ Calculated value \pm coefficient of variation \% } \\
\cline { 2 - 6 } & PHBV - 100\% & PLA - 100\% & iPP - 100\% & PHBV (70)/PLA (30) & PHBV (70)/iPP (30) \\
\cline { 2 - 6 } & C1 & C2 & C3 & C4 & C5 \\
\hline Breaking force, cN/tex & $7.60 \pm 6.13$ & $3.10 \pm 6.22$ & $18.40 \pm 4.92$ & $9.00 \pm 8.0$ & $8.80 \pm 7.99$ \\
\hline Elongation at break, \% & $22.70 \pm 5.35$ & $3.30 \pm 6.78$ & $292.70 \pm 2.66$ & $26.70 \pm 9.37$ & $125.40 \pm 7.82$ \\
\hline Work of break, J & $0.006 \pm 5.42$ & $0.005 \pm 5.46$ & $3.091 \pm 5.75$ & $0.098 \pm 8.26$ & $0.725 \pm 7.28$ \\
\hline Tensile modulus, cN/tex & $195.82 \pm 6.97$ & $39.31 \pm 7.83$ & $23.25 \pm 5.49$ & $106.03 \pm 8.15$ & $26.78 \pm 5.52$ \\
\hline
\end{tabular}

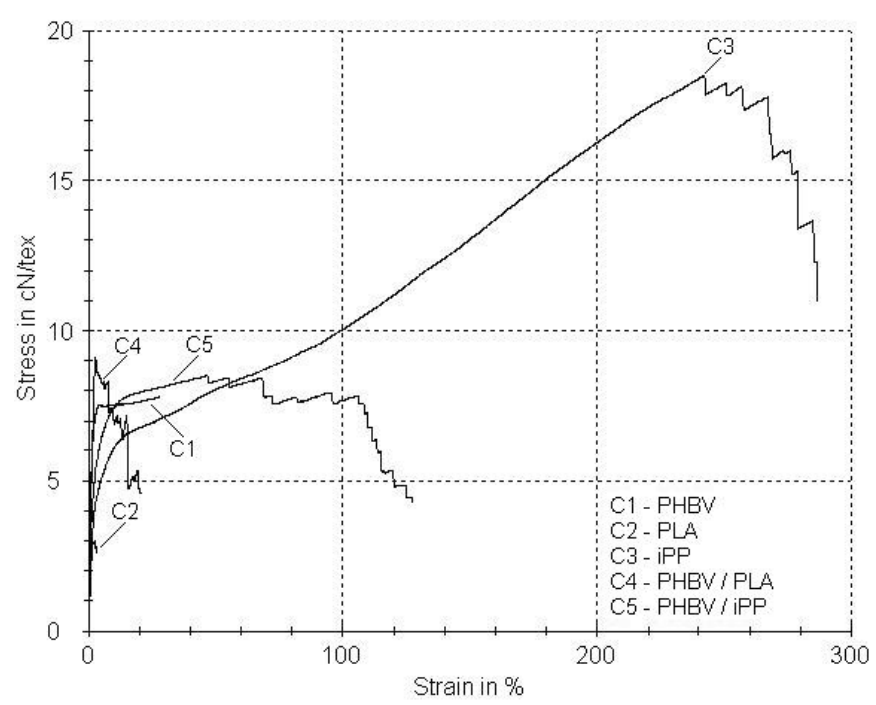

Figure 6. Tenacity-extension curve for yarns after destruction tests.

iPP is a paraffinic hydrocarbon, and so the absorption study showed that C3 yarns do not adsorb liquids as the other yarns. Since the iPP polymer is resistant to most liquids, sodium chloride is not an exception (Figure 5, C3). Throughout the 90 days of experiment, the $\mathrm{C} 3$ sample weight remained unchanged.

In general, C3 yarns show higher mechanical characteristics: breaking force increased insignificantly by $1.65 \%$, elongation at break increased by $4.65 \%$ and tensile modulus decreased only by $0.34 \%$. Comparing the $\mathrm{C} 3$ yarn mass with the initial point, the change dependence of destruction time described in the logarithmic equation, the result of the coefficient of the definition indicates $R^{2}=0$. This result shows that there is no correlation between the examined parameters and confirms that the sodium chloride $0.9 \%$ does not affect the mechanicalphysical properties of C3 (iPP) yarns. Extensive studies [39,40] testing the chemical stability of iPP when exposed to a lot of organic and inorganic chemicals has shown it to be highly stable against: acids, alkalise, aqueous solutions of inorganic salts, detergents, oils and greases, and gasoline, lubricants and other liquids.

Analysis of the kinetic curves shows that the maximum rate of weight loss is observed in the $\mathrm{C} 2$ sample among the yarn of pure polymers $(\mathrm{C} 1, \mathrm{C} 2$ and $\mathrm{C} 3)$. In the initial period of observation (14 days), the weight of the yarns has been changed by $4.61 \%$. The degradation of PLA is a function of several variables, among them temperature. Our results correspond with the results of other researchers $[26,41]$ where the onset of fragmentation occurs after about 9 days at $60^{\circ} \mathrm{C}$. The residual mass of $\mathrm{C} 2$ yarns was $81.13 \%$ after 90 days. The mechanical properties of the C2 yarns had considerably changed: breaking force decreased by $70.53 \%$, elongation at break deceased by $97.75 \%$ and tensile modulus decreased by $38.46 \%$. The dependence changes of destruction time are described in the logarithmic equation. The coefficient of the definition shows good connection of the $\mathrm{C} 2$ yarn results, $\mathrm{R}^{2}=$ 0.9981 . This result indicates that the relationship between the parameters is relatively strong.

The results of the experiment have suggested that the $\mathrm{C} 1$ (PHBV) samples degrades at a much slower rate and loses its properties at a slower rate compared with C2 (PLA) yarns. Weight loss hardly occurs within 50 days; during this period, the yarn loses $\sim 2.5 \%$ of weight. Marked gradual loss of weight begins after 60 days. The $\mathrm{C} 1$ yarns lost a smaller percentage of weight - $10.52 \%$ - after 90 days of incubation. Other authors [42,43] had described analogous results exploring the destruction of PHBV products, where the weight loss was 10 $20.2 \%$ during 83 days.

External condition of $\mathrm{C} 1$ yarns has not changed in 90 days after incubation. Significant defects on the yarn surface were not observed despite the significant decrease in weight $\left(R^{2}=\right.$ 0.9238). Small local defects like the filament abruption from the overall yarn structure were observed at a later date (70-90 days). The differences in weight loss primarily related to the conditions of solution desorption. It must be recalled that the structure of $\mathrm{C} 1$ yarn is monofilament [38] and the absorption of liquids of $\mathrm{C} 1$ samples was the lowest.

PHBV and PLA polymers are sufficiently biocompatible. During the degradation in the human body, PLA decomposes into lactic acid, which is naturally present in the human body as a result of metabolism. The hydrolytic degradation of PHB results in the formation of D-(-)-3-hydroxy-butyric acid, which is a normal constituent of blood (concentrations between 0.3 and $1.3 \mathrm{mM}$ ) $[26,43,44]$.

Analysis of the mass loss of blended C4 (PHBV/PLA) and C5 (PHBV/iPP) yarns showed that the initial stage of weight loss was different. Figure 5 shows that $\mathrm{C} 5$ yarn degrades at approximately the same speed as the yarn $\mathrm{C} 1$; the first signs of loss of weight can be seen only after 40 days whereas the 
sample C4 with $30 \%$ of PLA polymer degraded at a faster rate. After 40 days, the mass loss of C4 sample was already $5.26 \%$. Although the velocity of mass loss of $\mathrm{C} 4$ and $\mathrm{C} 1$ samples was different, after 90 days the residual yarns mass was the same at $89.47 \%$. This is $9.32 \%$ less than of C2. Ultimately, C5 yarn (with $30 \%$ of iPP polymer) lost $3.44 \%$ during the experiment. It is the smallest result, except for $\mathrm{C} 3$ yarns. The results also confirm the dependencies of investigated parameters, $\mathrm{R}^{2}=$ 9720 and $R^{2}=0.9238$ of $C 4$ and $C 5$ yarns, respectively.

Obviously, the degradation test lowers the values of mechanical parameters except for C3 (iPP) sample. In this case, the work of break parameter increased by $8.72 \%$ and the breaking force by $1.43 \%$ compared with C3 yarns before the destruction test. C2 samples showed the lowest mechanical characteristics of the results. The few or all mechanical characteristics of the yarns deteriorate over time. The same trend of changes in the mechanical characteristics of breaking force of the C4 and C5 samples was observed after 3 months of incubation in sodium chloride solution. C4 and C5 yarn samples decreased 12 and $5.17 \%$, respectively; the elongation at break and work of break decreased respectively for C4 by 21.92 and $58.82 \%$, and for C5 by 6.62 and $26.09 \%$, while the tensile modulus increased in both cases. Tensile modulus of $\mathrm{C} 4$ yarns increased by $3.21 \%$ and of C5 $1.94 \%$. Comparing blended C4 and C5 yarns, the smallest effect of sodium chloride solution of $0.9 \%$ was observed for the composition of PHBV/iPP 70/30 (C5). This is attributed to the fact that $30 \%$ of iPP in blended yarns inhibits the absorption of liquid.

\section{Conclusions}

This study has investigated the mechanical-physical properties of PHBV, PLA, iPP yarns and its PHBV/PLA and PHBV/iPP blends, which were prepared by extrusion equipment COLLIN CMF 100. The aim of the study was an attempt to improve the PHBV yarn structure and mechanical parameters by adding $30 \%$ of biodegradable PLA and non-degradable iPP polymers.

Although common processing techniques as extrusion can be used to fabricate PHBV into various yarn structures, its high sensitivity to degradation requires careful control of processing conditions. This study reconfirmed that it is impossible to produce multifilament structure of the pure PHBV polymer. The filaments stick together during the manufacturing process and result in a homogeneous yarn.

Blending $30 \%$ of PLA and iPP improved the elongation at break and tensile strength significantly while still keeping considerably high tensile modulus for the PHBV-rich blends. Also, the structure of pure PHBV yarns was improved. This amount of additional polymers allows exacting the multifilament structure of PHBV/PLA and PHBV/iPP yarns.

The PHBV/PLA and PHBV/iPP (70/30) blends show improved mechanical characteristics compared with pure PHBV and PLA yarns. The proportion of $30 \%$ of iPP and PLA reduced the stiffness and fragility of PHBV in blended yarns: breaking force improved to $\sim 42-74 \%$, yarns become more elastic 288$1426 \%$, and tensile modulus decreased $58-89 \%$.
Absorption analysis revealed that dynamic process of absorption depends on the structure and raw materials of the yarns. The disinfectant in all samples is absorbed faster than blood, because blood is a thick mixture of blood cells and plasma. The surface tension of disinfectant is $\sim 57 \%$ lower than blood. In both cases, PHBV yarn has good hydrophobic properties. The main reason could be that the structure of this yarn is not multifilament. The shortest absorption process was of pure PLA sample. As for iPP yarns, in our study the absorption process depends on the iPP yarn structure, which is multifilament, non-textured and without finish. In this case, the distribution of liquid occurs between the filaments, but does not soak into the filaments of the yarn. The process of disinfectant absorption in pure yarns goes on 79.25 until $1062 \mathrm{sec}$, the blood absorption in pure yarns goes on 201 until $3000 \mathrm{sec}$. Absorption time of blended yarns in disinfectant liquid case decreases to $87 \%$ and in case of blood absorption time increases to $795 \%$ compared with PLA and iPP yarns and decreases to $35 \%$ compared with $\mathrm{PHBV}$ yarns. This is a positive result given the fact that the yarn is focused on surgery field.

The degradation of yarns in sodium chloride solution was studied experimentally during 3 months. As observed in the biodegradation test, PHBV samples degrade at a much slower rate and lose their properties at a slower rate compared with PLA yarns. The PHBV yarns lost a smaller percentage of weight $-10.52 \%$ while pure PLA yarns lost about $\sim 18.87 \%$ of weight after 90 days of incubation. iPP yarns are not exposed to biodegradation since the threads do not absorb any liquids. PHBV/PLA yarns showed a more rapid and stable weight loss during all period $(10.52 \%)$ compared with PHBV/iPP yarns. Degradation of PHBV/iPP yarns is mainly due to destruction of PHBV component in blends. Therefore, destruction going on at the same rate as the PHBV sample and the loss of mass and mechanical characteristics changes are smaller compared with the $\mathrm{C} 4$ yarns.

The addition of PLA and iPP can be used to improve the tensile properties of PHBV compared with pure yarns. The right multifilament structure of PHBV/PLA and PHBV/iPP yarns with improved mechanical-physical characteristics was obtained as a result of research. Assessment of the PHBV/PLA and PHBV/ iPP yarns showed to be promising for use in medicine (surgical practice) as biodegradable and semi-degradable yarns since the polymers used are sufficiently biocompatible with the human body.

\section{References}

[1] Azizi, H.: Reactive Extrusion of Polypropylene: Production of Controlled - Rheology Polypropylene (CRPP) by Peroxide - Promoted Degradation, Polymer testing 23, pp. 137 - 143, 2004.

[2] Seregina, T. N.; Filbert, D. V.; et al.: The Properties of Polypropylene Yarn Modification with Medical Preparation, Research institute for synthetic fibers, No. 4, pp. $55-57$, 1974.

[3] Portnoy, R. C.: Polypropylene for Medical Application Medical Device Manufacturing and Technology, pp. 1 - 4, 2002. 
[4] Kolybaba, M.; Tabil, L. G; et al.: Biodegradable Polymers: Past, Present, and Future, The society for engineering in agricultural, food and biological systems, RRV03-0007, pp. 1 - 15, 2003.

[5] Asrar, J.; Gruys, K. J.: Biodegradable Polymer, Biodegradable polymer (Biopol), pp. $55-84$.

[6] Zinn, M.; Witholt, B.; Egli, T.: Occurrence, Synthesis and Medical Application of Bacterial Polyhydroxyalkanoate, Advanced drug delivery reviews, 53, pp. 5-21, 2001.

[7] Chun, Y. S.; Kim, W. N.: Thermal Properties of Poly (hydroxybutyrate - co - hydroxyvalerate) and Poly (e caprolactone) Blends, Polymer, 41, pp. 2305-2308, 2000.

[8] Spitalsky, Z.; Lacik, I. et al.: Controlled Degradation of Polyhydroxybutyrate via Alcoholysis with Ethylene Glycol or Glycerol, Polymer degradation and stability, 91, pp. 856 - 861, 2006.

[9] Ikada, J.; Tsuji, H.: Biodegradable polyesters for medical and ecological applications. Macromol. Rapid Commun. 21, pp. 117 - 132, 2000.

[10] Kohn, J.; Langer R. Bioresorbable and Bioerodible Materials in Biomaterials Science: An Introduction to Materials in Medicine, Ratner BD, Hoffman AS, Schoen FJ, and Lemons JE (eds), New York, Academic Press, pp. $64-72,1996$.

[11] Priming, M.: Tailoring the Properties of Bio - Based and Biocompostable Polymer Blends, ISBN: 978-90-386-25041, pp. $1-138$.

[12] Yu, L.; Dean, K.; Li, L.: Polymer Blends and Composites from Renewable Resources, Progress in polymer science, 31, pp. 576-602, 2006.

[13] Pachekoski, W. M.; Marcondes Agnelli, J. A.; Belem, L. P.: Thermal, Mechanical and Morphological Properties of Poly (hydroxybutyrate) and Polypropylene Blends After Processing, Materials research, Vol. 12, No. 2, pp. 159 - 164, 2009.

[14] Doi, Y.; Kanesawa, Y.; Tanahashi, N.: Biodegradation of Microbial Polyesters in the Marine Environment, Polymer degradation and stability, Vol. 36, Issue 2, pp. $173-177$, 1992.

[15] Bonartsev, A.; Boskhomdzhiev, A.; et al.: Degradation of Poly (3 - hydroxybutyrate) and its Derivatives: Characterization and Kinetic Behavior, Chemistry and chemical technology, Vol. 6, No. 4, pp. 385-392, 2012.

[16] Miller N.; Williams D.: On the Biodegradation of Poly - $\beta$ - hydroxybutyrate (PHB) Homopolymer and Poly - $\beta$ - Hydroxybutyrate - Hydroxyvalerate, Copolymers biomaterials, Vol. 8, 2. pp. 129 - 137, 1987.

[17] Galego, N.; Rozsa, C.; Sanchez, R.; Fung, J.; Vazquez, A.; Tomas, J. S.: Characterization and Application of Poly ( $\beta$-hydroxyalkanoates) Family as Composite Biomaterials, Polymer Testing, Vol. 19, pp. 485 - 492, 2000.

[18] Zinn, M.; Witholt, B.; Egli, T: Occurrence, Synthesis and Medical Application of Bacterial Polyhydroxyalkanoate, Advanced Drug Delivery Reviews, 53, p. 5-21, 2001.

[19] Maekawa, M.; Pearce, R.; Marchessault, R. H.; Manley, R. S. J.: Miscibility and Tensile Properties of Poly (beta hydroxy - butyrate) Cellulose Propionate Blends, Polymer, 40, pp. 1501 - 1505, 1999.

[20] Miao, M.; Qiu, Z.; et al.: Fully Biodegradable Poly (3 hydroxybutyrate - co - hydroxyvalerate) / Poly (ethylene succinate) Blends: Phase Behavior, Crystallizati on and Mechanical Properties, Reactive \& Functional Polymers, 68, pp. $446-457,2008$.
[21] Pivsa - Art, S.; Srisawat, N.; et al.: Preparation of Knitting Socks from Poly (lactic acid) and Poly [(R) - $3-$ hydroxybutyrate - co - (R) - 3- hydroxyvalerate] (PHBV) Blends for Textile Industrials, Energy Procedia, 9, pp. 589 - 597, 2011.

[22] Wang, S.; Ma, P.; et al.: Mechanical, Thermal and Degradation Properties of Poly (d,I - lactide) / Poly (hydroxybutyrate - co - hydroxyvalerate) / Poly (ethylene glycol) Blend, Polymer Degradation and Stability, 93, pp. 1364 - 1369, 2008.

[23] Yoon, J. S.; Lee, W. S.; Kim, K. S.; et al.: Effect of Poly (ethylene glycol) - block - Poly ( $L$ - lactide) on the Poly $[(R)$ - 3 - hydroxybutyrate] - Poly (L - lactide) Blends, European Polymer Journal, 36, pp. 435 - 442, 2000.

[24] Farrington, D. W.; Lunt, J.; Davies, S.; Blackburn, R. S.: Poly (lactic acid) Fibers, Biodegradable and Sustainable Fibres, pp. $191-220$.

[25] Hassan, A.; Balakrishnan, H.; Akbari, A.: Polylactic Acid Based Blends, Composites and Nanocomposites, Advances in Natural Polymers, DOI: 10.1007/978-3-64220940-6-11, pp. 361 - 396, 2013.

[26] Biodegradable Poly (lactic acid): Synthesis, Modification, Processing and Applications, Tsinghua University Press, Beijing and Springer - Verlag Berlin Heidelberg, pp. 142 207, 2010.

[27] Farrington, D. W.: Poly (lactic acid) Fibers, Biodegradable and Sustainable Fibres, Ingeo and Nature Works are Trademarks of Nature Works LLC, pp. 191 - 220.

[28] Bliimm E.; Owen, A. J.: Miscibility, Crystallization and Melting of Poly (3 - hydroxybutyrate) / Poly ( $L$ - lactide) Blends, Polymer, Vol. 36, No. 21, pp. 4077 - 4081, 1999.

[29] Koyama, N.; Doi, Y.: Miscibility of Binary Blends of Poly $[(R)-3-$ hydroxybutyric acid] and Poly [(S) - lactic acid], Polymer, Vol. 38, No. 7, pp. 1589 - 1593, 1997.

[30] Ohkoshia, H.; Abeb, Y.; Doib: Miscibility and Solid - State Structures for Blends of Poly [(S) - lactide] with Atactic Poly $[(R, S)-3$ - hydroxybutyrate], Polymer, 41, pp. 5985 - 5992, 2000.

[31] Focarete, M. L.; Ceccorulli, G.; Scandola, M.: Further Evidence of Crystallinity - Induced Biodegradation of Synthetic Atactic Poly (3 - hydroxybutyrate) by PHB Depolymerase a from Pseudomonas Lemoignei. Blends of Atactic Poly (3 - hydroxybutyrate) with Crystalline Polyesters Macromolecules, 31, pp. 8485 - 8492, 1998.

[32] Noda, I.; Satkowski, M. M.; Dowrey, A. A. et al.: Polymer Alloys of Nodax Copolymers and Poly (lactic acid), Macromolecular bioscience, 4, pp. 269 - 275, 2004.

[33] Zhang, M.; Thomas, N. L.: Blending Polylactic Acid with Polyhydroxybutyrate: The Effect on Thermal, Mechanical, and Biodegradation Properties, Advances in Polymer Technology, Vol. 30, No. 2, pp. $67-79,2011$.

[34] Gerard, T.; Budtova, T.: Morphology and Molten - State Rheology of Polylactide and Polyhydroxyalkanoate Blends, European polymer journal, 48, pp. 1110 - 1117, 2012.

[35] Krikštanavciene, K.; Stanys, S.; Jonaitiene, V.: Relation between Mathematically Simulated and Experimental Results of Polyhidroxybutyrate-co-valerate Yarns, Fibres \& Textiles in Eastern Europe, 21, 6(102), p. 27 - 32, 2013.

[36] Carroll, B. J.: The Accurate Measurement of Contact Angle, Phase Contact Areas, Drop Volume, and Laplace Excess Pressure in Drop on Fibre Systems, Journal of Colloid and Interface Science 57 (3), pp. 488 - 495, 1976. 
[37] Sodium Chloride Solution 0.9\% for Injection, Ukpar Sodium Chloride 0.9\% Solution for InjectionPL 08828/0178, p. 1- 15.

[38] Karahan, M.: Experimental Investigation of the Effect of Fabric Construction on Dynamic Water Absorption in Terry Fabrics, Fiber and Textiles in Eastern Europe, ISSN 1320 - 3666, Vol. 15, No. 3, pp. 74-80, 2007.

[39] Propex: The Durability of Polypropylene Geotextiles for Waste Containment Applications, Toxicology of Polypropylene, Matafaxx Vol. 2, No. 1, pp. 2-12, 1992.

[40] Mathur, A.; Netraval, N, A.: Chemical aging effects on the physio-mechanical properties of polyester and polypropylene geotextiles, Geotextiles and Geomembranes, Vol. 13, Issue 9, p. 591 - 626, 1994.
[41] Lakshmi, S. N.; Cato, T. L.: Biodegradable polymers as biomaterials, Progressing in Polymer Science, 32, pp. 762 - 798, 2007.

[42] Misra, S. K.; Valappil, S.; Roy, I.; Boccaccini, A. R.: Polyhydroxyalkanoate (PHA)/inorganic Phase Composites for Tissue Engineering Applications, Biomacromolecules, Vol. 7, No. 8, p. 2249 - 2258, 2006.

[43]Boschmdgiev. A. P. Study of Biodegradation of Biocompatible Polymer Systems Based on Polyhydroxyalkanoates, Biochemistry, pp. 1 - 28, 2010.

[44] Pathiraja, A.; Gunatillake, R.: Biodegradable Synthetic Polymers for Tissue Engineering, European Cells and Materials, ISSN 1473 - 2262, Vol. 5, pp. 1 - 16, 2003. 